\title{
MEDICIÓN DE LA EFICIENCIA RELATIVA DE FINCAS GANADERAS CON SERVICIO DE ASISTENCIA TÉCNICA*
}

\author{
ADRIANA LUCÍA CONTRERAS LEÓN"* \& GLORIA ISABEL RODRÍGUEZ LOZANO*** \\ UNIVERSIDAD NACIONAL DE COLOMBIA
}

Recibido/ Received/ Recebido: 04/03/2016-Aceptado/ Accepted / Aprovado: 04/07/2016

\begin{abstract}
Resumen
El objetivo de esta investigación fue medir la eficiencia relativa de fincas ganaderas a las cuales se les presta el servicio de asistencia técnica, ubicadas en los municipios de Duitama, Belén, Cerinza y Paipa en el departamento de Boyacá (Colombia). Para el desarrollo de esta investigación se usó la metodología Data Envelopment Analysis - DEA. La asistencia se realizó a 121 fincas, las cuales se convirtieron en la población objeto de esta investigación. A nivel de los resultados se evidenció que en más del treinta y cinco por ciento de las fincas el índice de eficiencia es superior al $60 \%$ y para el $25 \%$ de estas fincas su eficiencia es superior al $90 \%$.
\end{abstract}

Palabras clave: Medición de eficiencia; DEA; Explotación agrícola familiar; Eficiencia en fincas ganaderas.

\section{MEASUREMENT OF RELATIVE EFFICIENCY OF CATTLE FARMS WITH TECHNICAL SUPPORT}

\begin{abstract}
The objective of this research was to measure the relative efficiency of cattle farms that are provided with technical support. Located in the municipalities of Duitama, Belen, Cerinza and Paipa in the department of Boyaca (Colombia). For the development of this research Data Envelopment Analysis - DEA methodology was used. The technical support was performed at 121 farms which became the target population of this research. As a result it was shown that in more than thirtyfive percent of the farms the efficiency ratio is above $60 \%$ and for $25 \%$ of these farms efficiency is over $90 \%$.
\end{abstract}

Keywords: Efficiency measurement; DEA; Family farming activity; Efficiency in cattle farms.

Artículo de investigación científica derivado del proyecto: "Estudio de la eficiencia y la productividad de los sectores de la economía colombiana", perteneciente a la Universidad Nacional de Colombia.

Investigadora independiente. Candidata a Magister en Administración de la Universidad Nacional de Colombia, Administradora de Empresas Agropecuarias de la Universidad Pedagógica y Tecnológica de Colombia. Correo electrónico: alcontrerasl@unal.edu.co

*.* Profesora Asociada de la Facultad de Ciencias Económicas de la Universidad Nacional de Colombia. PhD en Ciencias Económicas de la Universidad Nacional de Colombia, Magister en Ingeniería Industrial de la Universidad de los Andes y Administradora de Empresas de la Universidad Nacional de Colombia. Dirección postal: Ciudad universitaria Bogotá, Edificio 311, Oficina 308. Teléfono: (57) 3165000 Ext. 12543. Correo electrónico: girodriguezl@unal.edu.co 


\title{
MEDIDA DA EFICIÊNCIA RELATIVA DE FAZENDAS DE GADO COM SERVIÇO DE ASSISTÊNCIA TÉCNICA
}

\begin{abstract}
Resumo
O objetivo desta pesquisa foi medir a eficiência relativa de fazendas de gado às quais se presta serviço de assistência técnica, localizadas nos municípios de Duitama, Belén, Cerinza e Paipa no departamento de Boyacá (Colômbia). Para o desenvolvimento desta pesquisa utilizou-se a metodologia Data Envelopment Analysis - DEA. A assistência realizou-se a 121 fazendas, as quais se tornaram a população objeto desta pesquisa. A nível dos resultados se evidenciou que em mais de trinta e cinco por cento das fazendas o índice de eficiência é superior a $60 \%$ e para $25 \%$ destas fazendas sua eficiência é superior a $90 \%$.

Palavras chave: Medida de eficiência; DEA; Exploração agrícola familiar; Eficiência em fincas ganadeiras.

Contreras, A. \& Rodríguez, M. (2017). Medición de la eficiencia relativa de fincas ganaderas con servicio de asistencia técnica. En: Revista de la Facultad de Ciencias Económica: Investigación y Reflexión. rev.fac.cienc.econ, XXV (1). DOI: http://dx.doi.org/10.18359/rfce.1776
\end{abstract}

JEL: Q16, D61, C67, D24.

\section{Introducción}

En el avance de las organizaciones y los sectores de la producción, el mejoramiento continuo y la eficiencia son dos de los más importantes aspectos que determinan su competitividad, facilitando su crecimiento y permanencia en las actuales condiciones de un mundo altamente dinámico, cambiante y globalizado. Para el sector agropecuario su desarrollo y el aumento de la competitividad pasa entre otros factores por su capacidad de innovación tecnológica y la asistencia técnica recibida por parte de diferentes instituciones tanto estatales como privadas. Para cumplir su propósito fundamental, estas instituciones deben tener tal nivel de eficiencia que garanticen la optimización de los recursos invertidos; parámetro que no siempre logra ser medido, dejando a estas instituciones con escasas posibilidades de plantear procesos de mejoramiento.

La asistencia técnica o extensión rural ha sido uno de los principales componentes de los programas de desarrollo del sector agropecuario en los últimos cincuenta años en América Latina, caracterizada por niveles de coordinación y articulación que no han logrado los impactos esperados en términos de cobertura y adopción tecnológica por parte de los sectores involucrados, así como la escasa profundidad de aspectos organizativos y comerciales que son vitales para el negocio agropecuario (Díaz, Borroto, Suárez \& Castillo, 2005, p. 410).

En Colombia son pocas las organizaciones que prestan servicios de asistencia técnica a sus agremiados, la organización que presta estos servicios a las fincas ganaderas objeto de esta investigación lo hace mediante un modelo propio que impulsa la formación participativa de grupos de mejoramiento ganadero donde se dinamiza las competencias técnicas, organizacionales y de innovación.

Para el desarrollo de esta investigación se usó la metodología Data Envelopment Analysis - DEA, que es definida como una herramienta efectiva para la medición de la eficiencia de decision making unit (DMU) con múltiples entradas y salidas (Emrouznejad, Anouze \& Thanassoulis, 2010, p. 298; Khodabakhshi \& Aryavash, 2014, p. 188). Para Cooper, Li, Seiford, Tone, Thrall \& Zhu (2001, p. 221) las mensionadas unidades son aquellas entidades (colegios, ban- 
cos, hospitales, empresas, etc.) que se consideran responsables de convertir las entradas en salidas. Adicionalmente porque permite comparar el desempeño relativo de un grupo de unidades que realizan procesos de transformación para producir bienes y/o generar servicios, utilizando el mismo tipo de recursos para producir un mismo tipo de productos; identificando la frontera eficiente y al mismo tiempo permitiendo hallar indicador único de eficiencia para las unidades estudiadas. Es un método que tiene un doble proceso de optimización, lo cual hace que sea muy confiable respecto a sus resultados (Coelli, 1998, p. 144). Incluso, las evaluaciones realizadas por este método corresponden a aproximaciones no paramétricas que se pueden utilizar para estimar las fronteras de producción y evaluar la eficiencia relativa de las unidades estudiadas (Cooper, Huang, Lelas, Li \& Olesen, 1998, p.61) En los últimos años, DEA se ha aplicado en el área de los servicios, por ejemplo buscando cuales áreas son las más eficientes (Zervopoulos \& Palaskas, 2011, p. 401), en el sector bancario (Najafi \& Aryanezhad, 2011, p. 273), en comercialización (Golden, Brockett, Betak, Smith \& Cooper, 2012, p. 2) y aplicación en la medición del desempeño en los juegos olímpicos (Lei, Li, Xie \& Liang, 2015, p. 379). Investigaciones en campo más afines al de esta investigación, se tiene el estudio en servicios a sistemas agrícolas en Portugal (Silva, Mendes \& Santos, 2012, p. 8)

En el caso específico de la ganadería, se tienen investigaciones en Venezuela (Urdaneta, Peña, González, Casanova, Cañas \& Dios-Palomares, 2010, p. 649; Peña, 2012, p. 5), Irán (Banaeian, 2011, p. 142), Irlanda (Kelly, Shalloo, Geary, Kinsella \& Wallace, 2012, p. 63), Jordania (Aldeseit, 2013, p. 37), Azores (Silva, Arzubi \& Berbel, 2012, p. 73), también en Azores la medición de la eficiencia del sistema de pastoreo de animales (Silva, Santos \& Mendes, 2012, p.83), medición de la importancia de las subvenciones en la eficiencia de las granjas lecheras (Silva \& Marote, 2012, p. 157). Concretamente para el caso Colombiano, se tiene una aplicación en fincas ubicadas en Guasca-Cundinamarca (Oviedo \& Rodríguez, 2011, p. 2616). Adicionalmente se tiene aplicación de DEA en el servicio de asistencia técnica a ganaderos en Pakistán (Javed, Khurshid, Hassan, Ali \& Nadeem, 2012, p. 145).
El objetivo de esta investigación fue medir la eficiencia relativa de fincas ganaderas a las cuales se les presta el servicio de asistencia técnica, ubicadas en los municipios de Duitama, Belén, Cerinza y Paipa en el departamento de Boyacá, en Colombia.

\section{Caracterización del servicio de asistencia técnica}

Buscando que la actividad ganadera contribuya a la conservación, recuperación y uso sostenible de la biodiversidad, a la vez que mejore sus indicadores productivos y de rentabilidad, la organización en cuestión desarrolló el Proyecto de Ganadería Colombiana Sostenible que tiene como propósito promover la adopción de sistemas de producción amigables con el medio ambiente, como lo son los sistemas silvopastoriles; para mejorar la gestión de los recursos naturales, incrementar la prestación de servicios ambientales y elevar la productividad en las fincas participantes. A través este proyecto se hace énfasis en la capacitación a productores y asistentes técnicos, en la provisión de asistencia técnica especializada para los productores, la promoción y apoyo para el acceso al crédito en la línea de sistemas silvopastoriles intensivos (SSPi) con o sin maderables e Incentivo para la Capitalización Rural (ICR).

La organización desde el año 2008, desarrolló una metodología para la prestación del servicio de asistencia técnica llamado Núcleos Municipales de Extensión y Mejoramiento para pequeños ganaderos, que incluye actividades de formación de adultos y asistencia técnica, con el fin de construir en conjunto (ganaderos y extensionistas) habilidades y conocimientos técnicos, ambientales, empresariales, sanitarios y económicos en ganadería; que generen innovación tecnológica y, de esta manera buscar mejorar la productividad de las empresas ganaderas. De la misma forma, construir redes sociales a través de actividades colectivas realizadas mediante equipos de cuatro ganaderos, denominados grupos de mejoramiento ganadero, los cuales a su vez conforman las llamadas unidades de atención, cada una de estas unidades está constituida por 14 grupos de mejoramiento ganadero, para un total de 56 ganaderos; los cuales están a cargo de un extensionista. 
Una vez están conformadas las unidades de atención se desarrolla la metodología, la cual consiste en brindar la asistencia técnica por ciclos, cada ciclo dura alrededor de 5 semanas; los dos primeros días del ciclo de atención cada extensionista es capacitado en un tema específico el cual va a replicar con sus ganaderos, el siguiente día atenderá dos grupos de mejoramiento ganadero, en los cuales el extensionista capacitará a los 4 ganaderos de cada grupo en el tema a tratar durante el ciclo. Los siguientes dos días realizará la visita personalizada en cada predio perteneciente a los dos primeros grupos de mejoramiento ganadero con una duración de dos horas cada visita, durante esta visita se refuerza el tema haciendo la aplicación práctica en cada predio; durante el día seis se realiza el monitoreo de las actividades realizadas por los extensionistas. Estas actividades se repiten durante las siguientes 4 semanas completando el ciclo de atención. Dos días durante el ciclo se dejan para imprevistos. Esta asistencia técnica prioriza la formación de los equipos regionales de campo en métodos de extensión rural, en técnicas de educación para adultos y en modelos de construcción del conocimiento a partir de las comunidades rurales.

Para la asistencia técnica a los ganaderos se emplean diferentes métodos de extensión y comunicación: capacitaciones, talleres, demostraciones de método, días de campo y visitas individuales. Estos métodos permiten al extensionista conocer de cerca al ganadero, a su familia y a su entorno, para de esta forma apoyar los procesos de cambio y que estos sean perdurables en el tiempo y redunden en el bienestar socioeconómico del productor y su familia.

El equipo de trabajo específico que brinda la asistencia técnica en Duitama, Cerinza, Belén y Paipa está conformado por un profesional operativo quien coordina las actividades de la regional; por un profesional de capacitación y logística, quien es el encargado de manejar las capacitaciones tanto de los extensionistas como de los ganaderos; por un profesional de monitoreo y evaluación, encargado de apoyar a la región en la gestión de la información desde el punto de vista técnico y administrativo; y finalmente por 3 extensionistas quienes son los en- cargados de realizar la asistencia técnica a los ganaderos y finalmente por un auxiliar operativa quien es el apoyo administrativo del equipo de trabajo.

Se han desarrollado seis ciclos de atención, los cuales se describen a continuación:

Conservación de forrajes: La temática tratada buscó dar alternativas alimenticias a los productores, que permitan enfrentar la crisis generada por la época seca a partir de la utilización y optimización de los recursos existentes en sus fincas. En el desarrollo de este ciclo se mostraron alternativas sencillas y de bajo costo para el ganadero, las cuales son de fácil adopción y adaptación, dado que no se requiere de grandes volúmenes de insumos ni de maquinaria para su elaboración.

Reproducción de Material Vegetal: Este ciclo de atención buscó aportar métodos y formas de construcción de viveros y semilleros locales, como una estrategia para el establecimiento de Sistemas Silvopastoriles. El objetivo del desarrollo de este ciclo, fue mostrar a los ganaderos que en su predio existen recursos arbóreos muy valiosos que pueden aprovechar o reproducir de manera sencilla y económica a través de la conformación de viveros.

Manejo de potreros: El objetivo de este ciclo de atención fue dar a conocer a los ganaderos el manejo adecuado de los potreros y las estrategias para incorporación de los árboles dentro de las fincas, lo cual permite mantener una alta producción de forraje de alta calidad durante un mayor período de tiempo, reduciendo la degradación, la erosión, el daño ambiental y la baja producción en su ganado.

Siembras de sistemas silvopastoriles: asistencia en la entrega de árboles a los productores interesados en el establecimiento de cercas vivas y árboles dispersos en potrero.

Fenómeno del niño: El objetivo principal de este ciclo fue dar información a los participantes sobre el fenómeno del niño, su origen, historia e impacto $y$ ofrecer al ganadero algunas alternativas como la cosecha de agua y conservación de forrajes. 
Abonos orgánicos: El objetivo principal fue dar a conocer a los ganaderos los abonos orgánicos como una alternativa efectiva, económica y de fácil preparación para sus cultivos.

\section{Metodología}

\subsection{Data Envelopment Analysis-DEA}

Es un método usado para estimar la eficiencia relativa, de una unidad que toma de decisiones (DMU) a partir de la obtención de una frontera eficiente, cuando se desconocen relaciones funcionales entre las entradas y las salidas que intervienen en un problema (Chediak, 2008, p. 31). No obstante lo anterior la medida se deriva de la medida universal de eficiencia: salida/entrada (Cooper, Seiford \& Tone, 2000 , p. 3). De otra parte, DEA es un modelo que se utiliza para evaluar la eficiencia relativa de $n$ DMU, mediante la resolución de problemas de programación lineal para cada unidad de acuerdo con los datos observados de entradas y salidas (Wei \& Yan, 2010, p. 612). Cuando se quiere establecer la eficiencia relativa, es importante entender que la eficiencia o ineficiencia de una determinada DMU está determinada tanto por su propio funcionamiento como por el funcionamiento de las otras, pues aun cuando el punto es que las DMU's seleccionadas sean homogéneas, cada una tiene características que la hacen única (Rodríguez \& Tarazona, 2015, p. 153). DEA ha experimentado un rápido desarrollo, convirtiéndose progresivamente en un conjunto de conceptos y metodologías, los cuales se han concretado en un abanico de modelos (Rodríguez, 2011, p. 156). Según Lee \& Saen (2012, p.220) DEA no requiere asumir previamente una función de producción para ser utilizado. Adicionalmente, esta metodología establece el valor de la ineficiencia (Cook, Kress \& Seiford, 1993, p. 135).

Los modelos tradicionales de la DEA proporcionan una clasificación categórica de la DMU en DMU eficientes e ineficientes (Chandrashekhar \& Banerjee, 2016, p. 223).Existen dos modelos principales en DEA: Constant Return to Scale CRS y Variable Return to Scale VRS (Quintanilha, Correia \& Angulo, 2012, p. 333). En el modelo CRS cualquier variación en las entradas produce una variación proporcional en las salidas, considerando retornos constantes de escala; por el contrario, el modelo VRS no asume proporcionalidad entre entradas y salidas, lo que permite retornos variables de escala (Cervera, Oviedo \& Pineda, 2013, p. 139). En el modelo VRS los retornos a escala pueden ser adicionalmente incrementales y decrementales (Lau, 2013, p. 599). Los dos modelos se pueden trabajar con orientación a las entradas o a las salidas. En la primera orientación se busca la máxima reducción en el nivel de entradas mientras permanece idéntico el nivel de producción. La otra orientación a las salidas busca el máximo incremento de las salidas sin modificar el nivel de las entradas. En este sentido una unidad no puede ser caracterizada como eficiente si es posible incrementar cualquier salida sin incrementar ninguna entrada y sin disminuir ninguna otra salida (Blasco \& Coll, 2006, p. 104). En este sentido, González \& Valdés (2009, p. 50) y Lee, Yu \& Wang (2012, p. 713) coinciden con que el primer desafío, al aplicar los modelos de DEA, consiste en seleccionar las variables que describan con mayor precisión el proceso de transformación de las DMU evaluadas. Otro aspecto a tener en cuenta para la aplicación de DEA, es que las DMU's seleccionadas deben cumplir con algunas condiciones: la primera de ellas es que deben ser homogéneas para que se puedan comparar y lo suficientemente heterogéneas para que se pueda extraer información de la comparación, otra condición es que debe tener alguna capacidad para gestionar los recursos de que disponen (Pino, Solís, Delgado \& Barea, 2010, p. 163).

\subsection{Diseño metodológico}

Teniendo en cuenta que la organización brinda asistencia técnica mediante asesoría y acompañamiento técnico gratuito a los productores rurales agropecuarios, pero que a la vez se desconoce cuáles y como han sido los efectos de esta asistencia técnica; se planteó medir la eficiencia de este servicio de asistencia abarcando el mayor número posible de fincas en los municipios de Duitama, Belén, Cerinza y Paipa en el Departamento de Boyacá. Se escogió esta zona teniendo en cuenta que la actividad ganadera es una de las principales actividades económicas que se desarrollan allí (Gobernación de Boyacá, 
2014, p. 20), señalando que la producción diaria de leche en el departamento es de 1'026.266 litros de leche, cifra que se ha mantenido estable en los últimos cuatro años, estando los cuatro municipios mencionados anteriormente entre los que hacen un mayor aporte a esta cantidad. En la actualidad la asistencia se realiza a 121 fincas, las cuales se convirtieron en la población objeto de esta investigación.

\subsection{Modelo DEA específico}

La eficiencia de las fincas ganaderas se midió con DEA, aplicando el modelo VRS con orientación a las salidas, buscando maximizar sus productos manteniendo constante los insumos ofrecidos; teniendo como base los argumentos expuestos por Coelli, Prasada, O'donell \& Battese (1998, p.180)

Para la aplicación del modelo se tomó como conjunto de DMU las 121 fincas atendidas en los municipios relacionados anteriormente entre diciembre del 2012 y diciembre de 2014.

De manera general se tienen $n$ DMU donde cada $\mathrm{DMU}_{\mathrm{i}}, \mathrm{j}=1,2, \ldots, n$, produce las mismas salidas en diferentes cantidades, $\mathrm{y}_{\mathrm{rj}}(\mathrm{r}=1,2,3, \ldots, \mathrm{s})$, utilizando las mismas $m$ entradas, $x_{i j}(i=1,2, \ldots, m)$, también en diferentes cantidades (Aparicio, Borras, Pastor \& Vidal, 2015, p.21).

La eficiencia de una $\mathrm{DMU}_{\mathrm{O}}$ específica, se evalúa con el modelo VRS orientada a las salidas como sigue:

$$
\max \phi+\varepsilon\left(\sum_{j=1}^{m} S_{j}^{-}+\sum_{r=1}^{s} S_{r}^{+}\right)
$$

Sujeto a:

$$
\begin{aligned}
& \sum_{j=1}^{n} X_{i j} \lambda_{j}+S_{i}^{-}=X_{i 0} \quad i=1,2, \ldots \ldots \ldots \ldots m ; \\
& \sum_{j=1}^{n} Y_{r j} \lambda_{j}-S_{r}^{+}=\phi Y_{r 0} \quad r=1,2, \ldots \ldots \ldots s ; \\
& \sum_{j=1}^{n} \lambda_{j} \quad=1 \\
& \lambda_{j}, S_{t}^{-}, S_{r}^{+} \geq 0 \quad \forall \mathrm{i}, \mathrm{r}, \mathrm{j}
\end{aligned}
$$

Donde $\varepsilon>0$ es un elemento no Arquimedeano definido menor que cualquier número real positivo.
Una explicación más detallada de los modelos básicos de DEA se encuentra en el capítulo escrito por Santos, Negas \& Santos (2012, p.37).

\subsection{Delimitación de entradas y salidas}

A continuación se definen las entradas y salidas para el modelo DEA teniendo en cuenta las características de cada ciclo de atención.

Las entradas fueron los insumos y recursos suministrados por la organización a cada una de las fincas

- Entrada 1: Horas de asistencia técnica: Son la dedicación en tiempo que el extensionista ha empleado para la capacitación y seguimiento a los temas de cada uno de los ciclos de atención.

- Entrada 2: Costo de capacitación: Hace referencia a los recursos financieros que destina la organización para el proceso de asistencia técnica en cada finca, representado en la nómina del equipo que interviene en dicha actividad.

- Entrada 3: Costo material vegetal: Es el valor monetario del material vegetal que recibe cada uno de las fincas ganaderas con destinación al establecimiento de sistemas silvopastoriles.

Como salidas (Tabla 1) se tuvieron en cuenta los productos de cada uno de los ciclos de atención descritos con anterioridad.

\section{Resultados}

Los resultados de eficiencia relativa muestran que las 121 DMU`s [fincas] evaluadas poseen diversos niveles y capacidades para la adopción y puesta en práctica del conjunto de recomendaciones tecnológicas, que en esta investigación se analizan como las entradas del sistema. El impacto de estas entradas se evalúa a partir de los resultados en términos productivos que muestran las fincas [evaluadas como las salidas del sistema], este comportamiento entre las entradas y salidas determina la eficiencia del sistema productivo de la finca.

Los resultados del índice de eficiencia de las 121 DMU`s realizado a través de DEA, se presentan en 
la Tabla 2, donde se indica la finca analizada y el índice obtenido en un rango que va desde $0.00 \%$ a 100.0\%, siendo este último el valor de máxima eficiencia posible para una DMU.

Tabla 1. Salidas

\begin{tabular}{|l|l|}
\hline \multicolumn{1}{|c|}{ Ciclo de atención (temas de capacitación) } & \multicolumn{1}{c|}{ Salida } \\
\hline Conservación de forrajes & Salida 1: Kilogramos de forraje conservado \\
\hline Reproducción de material vegetal & Salida 2: Número de árboles producidos \\
\hline Manejo de potreros & Salida 3: Número de hectáreas manejadas con división de potreros \\
\hline Siembras de sistemas silvopastoriles & Salida 4: Número de hectáreas establecidas con sistemas silvopastoriles \\
\hline Fenómeno del niño & Salida 5: Litros de agua almacenada \\
\hline Abonos orgánicos & Salida 6: Litros de abono orgánico producido \\
\hline
\end{tabular}

Fuente: Elaboración de las autoras.

Tabla 2. Índice de eficiencia modelo VRS orientación a las salidas

\begin{tabular}{|c|c|c|c|c|c|c|c|c|c|}
\hline DMU & Índice (\%) & DMU & Índice (\%) & DMU & Índice (\%) & DMU & Índice (\%) & DMU & Índice (\%) \\
\hline U1 & 30,76 & U26 & 100 & U51 & 68,26 & U76 & 40,43 & U101 & 99,3 \\
\hline U2 & 33,69 & U27 & 36,15 & U52 & 39,2 & U77 & 44,68 & U102 & 49,27 \\
\hline U3 & 36,23 & U28 & 100 & U53 & 23,97 & U78 & 27,66 & U103 & 98,1 \\
\hline U4 & 75,58 & U29 & 10,73 & U54 & 32,96 & U79 & 100 & U104 & 100 \\
\hline U5 & 38,93 & U30 & 18,19 & U55 & 42,35 & U80 & 60,92 & U105 & 100 \\
\hline U6 & 59,59 & U31 & 23,42 & U56 & 28,24 & U81 & 80,26 & U106 & 56,08 \\
\hline U7 & 37,98 & U32 & 20,47 & U57 & 89,39 & U82 & 15,69 & U107 & 26,03 \\
\hline U8 & 31,95 & U33 & 41,47 & U58 & 100 & U83 & 46,46 & U108 & 100 \\
\hline U9 & 45,33 & U34 & 9,9 & U59 & 96,97 & U84 & 15,69 & U109 & 87,14 \\
\hline U10 & 31,53 & U35 & 41,58 & U60 & 100 & U85 & 76,22 & U110 & 53,04 \\
\hline U11 & 23,24 & U36 & 65,49 & U61 & 89,14 & U86 & 40,58 & U111 & 61,52 \\
\hline U12 & 16,76 & U37 & 41,05 & U62 & 49,52 & U87 & 12,5 & U112 & 58,28 \\
\hline U13 & 45,35 & U38 & 29,34 & U63 & 72,73 & U88 & 69,45 & U113 & 50,65 \\
\hline U14 & 25 & U39 & 67,76 & U64 & 34,83 & U89 & 100 & U114 & 81,18 \\
\hline U15 & 45,23 & U40 & 100 & U65 & 73,2 & U90 & 42,7 & U115 & 54,46 \\
\hline U16 & 28,01 & U41 & 59,87 & U66 & 100 & U91 & 27,75 & U116 & 62,86 \\
\hline U17 & 23,2 & U42 & 32,12 & U67 & 42,41 & U92 & 77,2 & U117 & 100 \\
\hline U18 & 30,81 & U43 & 45,61 & U68 & 53,42 & U93 & 100 & U118 & 100 \\
\hline U19 & 36,3 & U44 & 31,68 & U69 & 100 & U94 & 77,32 & U119 & 100 \\
\hline U20 & 99,47 & U45 & 73,98 & U70 & 58,48 & U95 & 49,11 & U120 & 84,53 \\
\hline U21 & 18,38 & U46 & 42,5 & U71 & 25 & U96 & 52,19 & U121 & 100 \\
\hline U22 & 32,84 & U47 & 5,35 & U72 & 39,34 & U97 & 13,86 & & \\
\hline U23 & 46,7 & U48 & 44,99 & U73 & 100 & U98 & 38,24 & & \\
\hline U24 & 28,16 & U59 & 51,34 & U74 & 100 & U99 & 74,36 & & \\
\hline U25 & 100 & U50 & 100 & U75 & 45,11 & U100 & 13,76 & & \\
\hline
\end{tabular}

Fuente: Elaboración de las autoras. 
Para analizar el comportamiento del índice de eficiencia de las DMU`s, a continuación se estudian los diferentes niveles de eficiencia de las fincas evaluadas. En primera instancia los resultados evidencian que la eficiencia del servicio de asistencia técnica de la organización posee alto grado de satisfacción ya que en más del 35\% de las fincas el índice de eficiencia es superior al 60\%, y para del $25 \%$ de dichas DMU`s su eficiencia es superior al 90\% (Ilustración 1). Con lo anterior se infiere que las variables de entradas evaluadas inciden de manera positiva en su comportamiento productivo (medido a partir de las variables de salida) de las DMU`s analizadas.

De la misma forma se evidencia que el servicio de asistencia técnica de la organización logra altos niveles de impacto positivo. Lo anterior se confirma analizando los resultados que muestran que cerca del $2 \%$ de las DMU's poseen un bajo porcentaje de eficiencia inferiores al $10 \%$, indicando que solo en este porcentaje el servicio de asistencia técnica no logra influenciar de manera positiva o en bajo grado el desempeño de las DMU's evaluadas, de la misma forma los resultados muestran que cerca del 10\% de las DMU`s presentan valores de eficiencia entre el 11 y $20 \%$.

Para mayor comprensión de los resultados obtenidos a continuación se presenta el análisis detallado de las DMU`s que tienen comportamientos diferen- ciados en su nivel de eficiencia, identificando con ello las posibles causas que puedan incidir en dicho indicador. En las DMU's analizadas cerca del 17\% (21 DMU`s) poseen un porcentaje de eficiencia del $100 \%$. Lo anterior se explica a partir del comportamiento de la variable de entrada 3 (costo de material vegetal) que en estas DMU's posee los valores más altos, ya que la cantidad de material vegetal adquirido es superior a las demás DMU's, dicho comportamiento es directamente proporcional y se refleja en la variable de salida 4 (área establecida en sistemas silvopastoriles), donde estas unidades presentan en forma concordante valores por encima de la media de todas las unidades evaluadas. En igual sentido lo anterior se evidencia en el comportamiento de la variable de salida 1 (kilogramos de forraje conservado) donde la mayoría de estas DMU`s presentan valores que están por encima del resto de dichas unidades.

Para demostrar lo anterior se establece el coeficiente de correlación entre los valores del índice de eficiencia calculado y los valores de las variables de salida (Tabla 3), los resultados muestran que la mayor correlación se da con la variable de salida 1 (kilogramos de forraje conservado) con un valor de 0.52 (alta fuerza de correlación), indicando que estos resultados de eficiencia de las DMU son altamente consistentes con la cantidad de forraje producido, igualmente el coeficiente de correlación es alto (0.5) para la variable de

Ilustración 1. Distribución en porcentaje eficiencia de las DMU

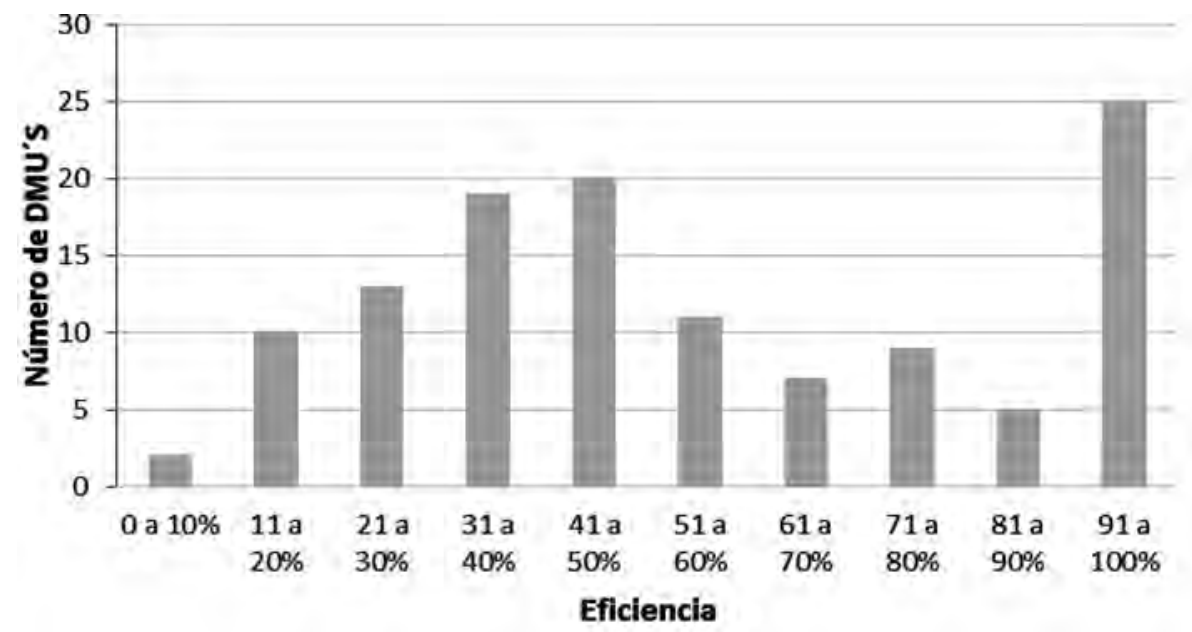

Fuente: Elaboración de las autoras. 
Tabla 3. Índices de correlación entre variables de salida e índice de eficiencia

\begin{tabular}{|l|c|c|}
\hline Salida $\mathrm{x}$ eficiencia & Correlación & $\begin{array}{c}\text { Fuerza de la } \\
\text { correlación }\end{array}$ \\
\hline Salida 1 Kilogramos de forraje conservado x eficiencia & $\mathbf{0 , 5 2}$ & Alta \\
\hline Salida2 Número de árboles producidos x eficiencia & 0,45 & Media \\
\hline Salida3 Número de hectáreas manejadas con división de potreros x eficiencia & $\mathbf{0 , 5 0}$ & Alta \\
\hline Salida4 Número de hectáreas establecidas con sistemas silvopastoriles x eficiencia & 0,42 & Media \\
\hline Salida5 Litros de agua almacenada x eficiencia & 0,30 & Media \\
\hline Salida6 Litros de abono orgánico producido x eficiencia & 0,42 & Media \\
\hline
\end{tabular}

Fuente: Elaboración de las autoras.

salida 3 (área implementada en división de potreros) que también tiene alta fuerza de correlación. Por el contrario la variable de salida 5 (cantidad de agua almacenada) presenta el coeficiente de correlación más bajo con un valor de 0.3 que indica que los resultados de esta variable no inciden significativamente en la conformación del índice de eficiencia de las DMU's.

El comportamiento destacado del grupo de veintiún (21) DMU's que presentan el índice de eficiencia más alto $(100 \%)$ obedece a su capacidad para apropiar e implementar la tecnología y las prácticas productivas que le son transferidas mediante la prestación del servicio de asistencia técnica que ofrece la organización. En este comportamiento es importante destacar que las variables que tienen fuerte incidencia para lograr el mayor índice de eficiencia son: kilogramos de forraje conservado y área implementada en división de potreros, indicando con lo anterior que son las aspectos que deben ser tenidos en consideración tanto por el servicios de asistencia técnica como por los propios ganaderos para mejorar los aspectos productivos y por ende la eficiencia del sistema de producción de las DMU's. Otra importante característica de estas unidades es que recibieron la menor cantidad de horas de asistencia técnica, y por el contrario recibieron la mayor cantidad de material vegetal con destino a la implementación o fortalecimiento del sistema silvopastoril.

En los resultados es importante mencionar el comportamiento atípico de dos (2) DMU`s (U 47, y U 34) que presentan valores de $5.3 \%$ y $9.9 \%$ de eficiencia respectivamente, estos resultados son extremadamente inferiores al promedio de eficiencia de las
DMU's analizadas que es de 55.2\%. Lo anterior se da por la baja adopción por parte de los ganaderos de los temas tratados en los ciclos de atención (entradas), imposibilitando mayores niveles de participación e interacción con el servicio de asistencia técnica.

En general las DMU's poseen alto grado de dispersión en su índice de eficiencia, dificultando el análisis comparativo de las mismas y limitando la posibilidad de encontrar las fuentes generadoras de estos valores. Para superar esta limitante se propone hacer un análisis teniendo como factor agrupante, el tamaño de las DMU's. Para lo anterior se organizan tres grupos a saber: DMU's pequeñas de área inferior a 10 hectáreas, medianas que poseen áreas entre 10 y 20 hectáreas y DMU's grandes que poseen área superior a 20 hectáreas.

En la Ilustración 2 se observa que en el grupo de DMU's grandes, el 56\% (14 fincas) se ubican en un índice de eficiencia mayor al 80\%, esto se relaciona con la alta fuerza de correlación de la salida 1(Kilogramos de forraje conservado) y salida 3 (Número de hectáreas manejadas con división de potreros), lo anterior indica que estas DMU's poseen ventajas comparativas por poseer un área mayor para el proceso productivo de la finca con lo cual se produce mayor cantidad de forraje conservado, dividir los potreros optimizando la relación días de descanso/días de ocupación de las praderas con lo cual se logra un efecto positivo en la calidad y cantidad de alimentación para los animales ya que la producción continua de forraje permite disminuir la estacionalidad en la producción de leche y/o carne en las DMU's. 
La DMU (U48) considerada como grande (más de 20 ha.) que se ubica en una eficiencia menor al 10\% ha producido salidas por encima del promedio grupal (número de árboles producidos, área implementada en división de potreros, área establecida en SSP, cantidad de agua almacenada y litros de abono orgánico producidos) pero los insumos (entradas) han sido considerablemente altos para estos resultados. El grado de implementación de las tecnologías ha sido baja de acuerdo a la alta capacitación recibida por parte del productor.

El grupo de los predios medianos (10 - 20 hectáreas) tiene una distribución normal en su índice de eficiencia, nueve (9) DMU's presentan una eficiencia superior al 90\%, las cuales se caracterizan por haber recibido mayor cantidad de material vegetal que genera una mejor respuesta en el establecimiento de SSP y conservación de forraje, por lo tanto se logra mayor índice de eficiencia.

Con respecto a las DMU's pequeñas (menor a 10 hectáreas) cerca de 37 fincas que corresponden al $79 \%$ tienen un índice de eficiencia inferior al 50\%, en términos relativos este grupo es el que presenta menor eficiencia y por lo tanto menor impacto del servicio de asistencia técnica. No se asume su tamaño como predictor de este resultado, pues existen cinco (5) DMU's pequeñas con eficiencia mayor al 90\%. El grupo de DMU's fincas pequeñas y eficientes se caracterizan por optimizar al máximo las horas de asistencia técnica ofrecidas por el extensionista y por optimizar elaboración de abonos orgánicos.

\section{Conclusiones y recomendaciones}

Se identificaron las unidades que por su eficiencia se convierten en referentes para aquellas que con condiciones similares en cantidad de entradas poseen niveles de eficiencia inferior, cada grupo de referentes constituye la base para construir e implementar un sistema de mejora continua donde las unidades ineficientes logren mejorar los niveles de eficiencia. En la Tabla 4 se muestra las unidades que sirven como referenciales para las DMU's ineficientes dentro de cada grupo (grandes, pequeñas, medianas).

Tabla 4. Grupos de referencia

\begin{tabular}{|l|l|}
\hline Grupos DMU's & \multicolumn{1}{|c|}{ Referenciales } \\
\hline Pequeñas & U40, U105,U117,U121 \\
\hline Medianas & U26,U28,U50,U60,U73,U93,118 \\
\hline Grandes & $\begin{array}{l}\text { U25,U58,U66,U69,U74,U79,U89, } \\
\text { U104,U108,U119 }\end{array}$ \\
\hline
\end{tabular}

Fuente: Elaboración de las autoras.

Ilustración 2. Eficiencia de DMU's agrupadas por tamaño

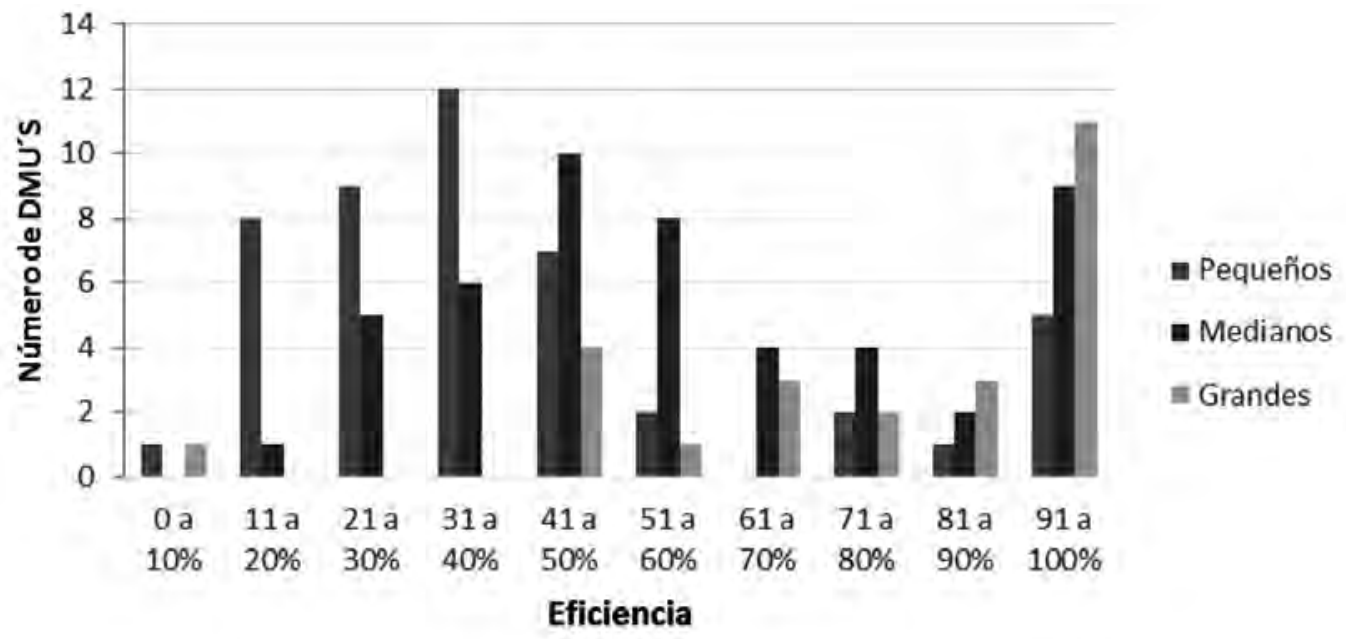

Fuente: Elaboración de las autoras. 
Para el grupo de las pequeñas existen 4 unidades que les sirven como referentes; de la misma forma para el grupo de las unidades medianas posee 7 fincas que cumplen dicha condición; para las DMU`s grandes son 10 las unidades referenciales. Los resultados de la Tabla 4 indican que para el caso de las unidades grandes el $40 \%$ de ellas son consideradas como referentes del resto de su grupo; mientras que para el grupo de las medianas las unidades referentes son el $14.3 \%$ y en el grupo de las pequeñas solamente el $8.5 \%$ son unidades referentes. Con lo anterior es posible afirmar que el modelo de asistencia técnica de la organización logra mejores resultados en unidades consideradas como grandes, por el contrario para las unidades del grupo de medianas y pequeñas el modelo de asistencia técnica no posee resultados significativos.

De tal manera es posible afirmar que es necesario diseñar un modelo de asistencia técnica que responda a las necesidades y expectativas de las unidades pequeñas y medianas. Priorizando las actividades que realizan las fincas pequeñas y medianas que logran ser eficientes, para el caso de las fincas pequeñas la salida 6 (litros de abono orgánico producidos) que por su bajo costo de realización y el uso de insumos procedentes de las mismas fincas, su implementación no generará costos adicionales y por el contrario repercuten en los niveles de eficiencia. Para el caso de las fincas medianas se debe hacer énfasis en la salida 4 (número de hectáreas establecidas en sistemas silvopastoriles) y la salida 2 (número de árboles producidos) ya que con los resultados obtenidos se ha demostrado que tal actividad logra buen desempeño en este tipo de fincas en cuanto a la eficiencia.

Con los resultados obtenidos en esta investigación se recomienda que la organización ajuste su modelo de asistencia técnica para lograr que las fincas sean eficientes en el desarrollo del mismo, consiguiendo que todas las fincas adopten las tecnologías transferidas y recomendaciones dadas a través de sus extensionistas. A continuación se presentan las acciones que se propone sean emprendidas por la organización para optimizar su servicio de asistencia técnica, y de esta manera las fincas puedan interiorizar las mejoras:
- Por la alta heterogeneidad de las fincas ganaderas que reciben asistencia técnica es necesario hacer un proceso inicial de tipificación de dichas fincas, de acuerdo a su tamaño para aplicar un proceso diferencial en la asistencia técnica.

- El modelo de asistencia técnica debe conformar los grupos de mejoramiento ganadero teniendo en cuenta las DMU's identificadas como referenciales en la aplicación del modelo DEA, que permitirá un mayor nivel de adopción tecnológica ya que las referenciales serán el modelo a seguir para su grupo.

- Se debe realizar la medición de la eficiencia de manera sistemática y sostenida en el tiempo, para lograr que el mejoramiento en las prácticas sea sostenido.

\section{Referencias}

Aldeseit, B. (2013). Measurement of scale efficiency in dairy farms: data envelopment analysis (DEA) Approach. $J$ Agric Sci, 5(9): 37-43.

Aparicio, J., Borras, F., Pastor, J. \& Vidal, F. (2015). Measuring and decomposing firm's revenue and cost efficiency: The Russell measures revisited. Int. J. Production Economics, 165: 19-28

Banaeian, N. (2011). Do the Cattle Farms of Iran Produce Economically Efficient or Not? Asian Journal of Agricultural Sciences, 3(2): 142-149.

Blasco, O. \& Coll, V. (2006). Evaluación de la eficiencia mediante el análisis envolvente de datos: Introducción a los modelos básicos. España: Editorial Eumed.

Cervera, A., Oviedo, W. \& Pineda, J. (2013). Revisión bibliográfica de la aplicación de la metodología DEA en el ámbito educativo colombiano. Revista Civilizar Ciencias Sociales y Humanas, 13(25): 133-156.

Chandrashekhar, K. \& Banerjee, P. (2016). Measuring and benchmarking managerial efficiency of project execution schedule performance. International Journal of Project Management, 34: 219-236.

Chediak, F. (2008). La técnica DEA para medir y analizar la eficiencia, municipios del departamento del Tolima. Tesis doctoral inédita no publicada. Pereira: Universidad Tecnológica de Pereira, Facultad de Ingeniería Industrial.

Coelli, T. (1998). A multi-stage methodology for the solution of orientated DEA models. En: Operations Research Letters, 23: $143-149$

Coelli, T., Prasada, D., O'donell, C. \& Battese, G. (1998). An introduction to efficiency and productivity analysis. Segunda Edición Springer UEA. 
Cook W., Kress, M. \& Seiford, L. (1993). On the use of ordinal data in data envelopment analysis. En: Journal of Operational Research Society, 44, 133-140.

Cooper, W.W., Huang, Z.M., Lelas, V., Li S.X. \& Olesen,O.B. (1998) Chamce Constrained Programming Formulations for Stchocasting Characterizations of Efficiency and Dominance in DEA. En: The Journal of Productivity Analysis, 9: 53-79.

Cooper, W. W., Seiford, L. M. \& Tone, K. (2000). Data Envelopment Analysis: A Comprehensive Text with Models, Applications, References and DEA-Solver Software. Boston, Massachusetts: Kluwer Academic Publishers

Cooper, W. W., Li, S., Seiford, L. M., Tone, K., Thrall, R. M. \& Zhu, J. (2001). Sensitivity and Stability Analysis in DEA: Some Recent Developments. En: Journal of Productivity Analysis, 15: 217-246.

Díaz, J., Borroto, O., Suárez, J. \& Castillo, E. (2005) La transferencia de tecnologías en el sector ganadero latinoamericano. La experiencia cubana. En: Revista Cubana de Ciencia Agrícola, 39: 407-414.

Emrouznejad, A., Anouze, A. \& Thanassoulis, E. (2010) A semioriented radial measure for measuring the efficiency of decision making units with negative data, using DEA. En: European Journal of Operational Research, 200(1): 297-304.

Gobernación de Boyacá. (2014) Observatorio Económico de Boyacá.

Golden, L., Brockett, P., Betak, J., Smith, K. \& Cooper, W. (2012) Efficiency metrics for nonprofit marketing/fundraising and service provision-a DEA analysis. En: JMMR, 10: 1-25.

González, M. \& Valdés, N. (2009) Método de selección de variables para mejorar la discriminación en el análisis de eficiencia aplicando modelos DEA. En: Ingeniería Industrial, 8 (2): 45 - 56.

Javed, M., Khurshid, W., Hassan, I., Ali, A. \& Nadeem, N. (2012) Impact of institutional credit and extension services on productive efficiency of farms: Evidence from irrigated Punjab, Pakistan. En: J. Agric. Res, 50(1): 145-153.

Kelly, E., Shalloo, L., Geary, U, . Kinsella, A. \& Wallace M. (2012) Application of data envelopment analysis to measure technical efficiency on a sample of Irish dairy farms. En: Irish Journal of Agricultural and Food Research, 63-77.

Khodabakhshi, M. \& Aryavash, K. (2014) The fair allocation of common fixed cost or revenue using DEA concept. En: Annals of Operations Research, 214(1): 187-194.

Lau, K. (2013) Measuring distribution nefficiency of a retail network through data Envelopment analysis. En: Int. J. Production Economics, 146: 598-611.

Lee, G., Yu, M. \& Wang, L. (2012) DEA-based integrated relationship of returns to scale -an application to road maintenance in Taiwan. En: J Civ Eng Manag, 18(5): 709-723.

Lee, K. \& Saen, R. (2012) "Measuring corporates us tainability management: A data envelopment analysis approach". En: Int. J. Production Economics, 40: 219-226

Lei, X., Li, Y., Xie, Q. \& Liang, L. (2015) Measuring Olympics achievements based on a parallel DEA approach. En: Ann Oper Res, 226:379-396

Najafi, E. \& Aryanezhad, M. (2011) A BSC-DEA approach to measure the relative efficiency of service industry: A case study of banking sector. En: International Journal of Industrial Engineering Computations, 2(2): 273-282.

Oviedo, W. \& Rodríguez, G. (2011) Medición de la eficiencia técnica relativa de las fincas asociadas a Coounión en Guasca Cundinamarca. En: Rev MVZ Córdoba, 16(2): 2616-2627.

Peña, M. (2012). Análisis de la gestión empresarial en bovinos doble propósito y su relación con la eficiencia técnica. Caso Municipios Catatumbo y Colón, Estado Zulia. Tesis doctoral inédita no publicada. Córdoba: Universidad de Córdoba, Escuela Técnica Superior de Ingenieros Agrónomos y de Montes.

Pino, J., Solís, F., Delgado, M. \& Barea, R. (2010) Evaluación de la eficiencia de grupos de investigación mediante análisis envolvente de datos (DEA). En: El profesional de la información, 19 (2): 160-167.

Quintanilha, J., Correia, J. \& Angulo, L. (2012) Evaluación de la eficiencia de las compañías aéreas brasileñas a través de un modelo híbrido de análisis envolvente de datos (DEA) y programación lineal multiobjetivo. En: Ingeniare. Revista chilena de ingeniería, 20 (3): 331-342.

Rodríguez, G. \& Tarazona, O. (2015) Unidades de emprendimiento bajo un estudio de eficiencia relativa. En: Rev fac cienc econ, XXIII (2): 149-162.

Rodríguez, G. (2011). Indicadores DEA (Data Envelopment Analysis) de eficiencia y productividad para las actividades de extensión universitaria. Aplicación en la Universidad Nacional de Colombia. Bogotá: Centro Editorial Facultad de Ciencias Económicas Universidad Nacional de Colombia.

Silva, E., Arzubi, A. \& Berbel,J. (2012) An Application of Data Envelopment Analysis (DEA) in Azores Dairy Farms. Capítulo 5. Efficiency Measures in the Agricultural Sector, with aplications. Springer Netherlands

Silva, E. \& Marote, E. (2012) The Importance of Subsidies in Azorean Dairy Farms' Efficiency. Capítulo 11. Efficiency Measures in the Agricultural Sector, with aplications. Springer Netherlands.

Silva, E., Mendes, A, \& Santos, J. (2012) Efficiency Measures in the Agricultural Sector: The beginning. Capitulo 1. Efficiency Measures in the Agricultural Sector, with aplications. Springer Netherlands.

Silva, E., Santos, C. \& Mendes, A. (2012) Animal Grazing System Efficiency. Capitulo 6. Efficiency Measures in the Agricultural Sector, with aplications. Springer Netherlands.

Santos, J., Negas E. \& Santos L. (2012) Introduction to Data Envelopment Analysis. Capitulo 3. Efficiency Measures in the Agricultural Sector, with aplications. Springer Netherlands.

Urdaneta, F., Peña, M., González, B., Casanova, Á., Cañas, J. \& Dios-Palomares, R. (2010) Eficiencia técnica en fincas ganaderas de doble propósito en la cuenca del lago de Maracaibo, Venezuela. En: Revista Científica, 20(6): 649-658.

Wei, Q. \& Yan, H. (2010) A Data Envelopment Analysis (DEA) evaluation method based on sample decision making units. En: Int J Inf Technol Decis Mak, 9 (4): 601-624.

Zervopoulos, P. \& Palaskas, T. (2011) Applying quality-driven, efficiency-adjusted DEA (QE-DEA) in the pursuit of highefficiency-high-quality service units: an input-oriented approach. En: IMA, 22(4): 401-417. 